PROCEEDINGS OF THE

AMERICAN MATHEMATICAL SOCIETY

Volume 138, Number 5, May 2010, Pages 1581-1585

S 0002-9939(09)10153-3

Article electronically published on December 21, 2009

\title{
GENERALIZED AUSLANDER-REITEN CONJECTURE AND TILTING EQUIVALENCES
}

\author{
JIAQUN WEI
}

(Communicated by Birge Huisgen-Zimmermann)

\begin{abstract}
We show that the generalized Auslander-Reiten conjecture is stable under tilting equivalences.
\end{abstract}

\section{INTRODUCTION}

Throughout this paper, we consider Artin algebras and finitely generated left modules over them. Let $R$ be an Artin algebra. We denote by $\bmod R$ the category of all finitely generated left $R$-modules. For an $X \in \bmod R$, we denote by $\operatorname{pd} X$ (id $X$, resp.) the projective (injective, resp.) dimension of $X$. We denote by $R^{o}$ the opposite algebra and an $R^{o}$-module $M$ means the right $R$-module $M_{R}$.

In studying the Nakayama conjecture, Auslander and Reiten [1] proposed the following conjecture.

Auslander-Reiten conjecture. Let $R$ be an Artin algebra and let $X$ be an $R$ module. If $\operatorname{Ext}_{R}^{i}(X, X \oplus R)=0$ for all $i \geq 1$, then $X$ is projective.

Auslander and Reiten [1] proved the conjecture over Artin algebras such that every module $M$ has an ultimately closed projective resolution, that is, there is some syzygy $N$ of $M$ such that all indecomposable direct summands of $N$ already appear in earlier syzygies. This includes algebras of finite representation type, algebras with radical square zero and all torsionless-finite algebras.

We note that the Auslander-Reiten conjecture is related to the little finitistic dimension conjecture for Artin algebras, i.e., that $\operatorname{fdim} R=\sup \left\{\operatorname{pd}_{R} X \mid \operatorname{pd}_{R} X<\infty\right\}$ is always finite for an Artin algebra $R$. More precisely, if the finitistic dimension conjecture holds for all Artin algebras, then the Auslander-Reiten conjecture holds for all Artin algebras. However, for an Artin algebra satisfying the finitistic dimension conjecture, we don't know if it also satisfies the Auslander-Reiten conjecture. In fact, if we restrict $R$ to be self-injective, then the Auslander-Reiten conjecture is just the Tachikawa conjecture [7, which is still open up to now.

In this paper, we consider a generalized version of the Auslander-Reiten conjecture, which is stated as follows.

Received by the editors March 29, 2009, and, in revised form, June 20, 2009, and July 23, 2009 .

2000 Mathematics Subject Classification. Primary 16E10, 16E30, 16E65.

Key words and phrases. Auslander-Reiten conjecture tilting equivalence.

The author is supported by the National Science Foundation of China (Grants No. 10601024 and 10971099) and partially by the Jiangsu Natural Science Foundation (Grant No. BK2007220). 
Conjecture. Let $X$ be an $R$-module and $t$ be a nonnegative integer. If $\operatorname{Ext}_{R}^{i}(X$, $X \oplus R)=0$ for all $i>t$, then $\operatorname{pd}_{R} X \leq t$.

Obviously, in case $t=0$ we restate the Auslander-Reiten conjecture. It was shown in 9] that the generalized Auslander-Reiten conjecture holds for Artin algebras with every module having an ultimately closed projective resolution. In fact, to our knowledge, it holds for all algebras that are known to satisfy the AuslanderReiten conjecture.

It is still not known whether the generalized Auslander-Reiten conjecture holds for all Artin algebras if the finitistic dimension conjecture holds for all Artin algebras. Neither do we know if the Auslander-Reiten conjecture implies the generalized Auslander-Reiten conjecture.

Our aim in this paper is to show that the generalized Auslander-Reiten conjecture is stable under tilting equivalences, as was done by Happel for the finitistic dimension conjecture [5]. More precisely, we prove that if $T$ is a tilting or cotilting module over an Artin algebra $R$ with $S=\left(\operatorname{End}_{R} T\right)^{\circ}$, then $R$ satisfies the generalized Auslander-Reiten conjecture if and only if so does $S$. The result is presented in Section 3. In Section 2 we introduce the notions and recall the basic knowledge of the tilting theory which will be used in the proof.

We note that recently it was shown that the finitistic dimension conjecture is stable under derived equivalences [4. It would be interesting to consider the stability of the generalized Auslander-Reiten conjecture under derived equivalences.

\section{Preliminaries}

Let $R$ be an Artin algebra and $M \in \bmod R$. We denote by $\operatorname{add}_{R} M$ the category of modules isomorphic to direct summands of finite direct sums of $M$. The notion $M^{\perp}$ denotes the category of all modules $N \in \bmod R$ such that $\operatorname{Ext}_{A}^{i \geq 1}(M, N)=0$. Dually, the notion ${ }^{\perp} M$ denotes the category of all modules $N$ such that $\operatorname{Ext}_{A}^{i \geq 1}(N, M)$ $=0$. We denote by $M \gg 0$ the category of all modules $N$ satisfying that there is some integer $t \geq 0$ such that $\operatorname{Ext}_{R}^{i}(M, N)=0$ for all $i>t$, and we denote by $\hat{M}$ the category of all $R$-modules $N$ such that there is an exact sequence $0 \rightarrow C_{m} \rightarrow \cdots \rightarrow C_{0} \rightarrow N \rightarrow 0$ for some nonnegative integer $m$, where each $C_{i} \in \operatorname{add}_{R} M$.

We denote by $\mathbf{D}$ the usual duality functor between $\bmod R$ and $\bmod R^{o}$. For an $M \in \bmod R$ and a positive integer $t, \Omega_{R}^{t} M$ denotes the $t$-th syzygy of $M$.

We recall now some basic knowledge of the tilting theory. The reader is referred to 2, 3, 6] for complete proofs.

Let $R$ be an Artin algebra and $n$ be a nonnegative integer. Recall that $T \in \bmod R$ is called a tilting module of projective dimension at most $n$ if it satisfies

(T1) $\operatorname{pd} T \leq n$; i.e., there is an exact sequence $0 \rightarrow P_{n} \rightarrow \cdots \rightarrow P_{0} \rightarrow T \rightarrow 0$ with each $P_{i}$ projective.

(T2) $\operatorname{Ext}_{R}^{i}(T, T)=0$ for all $i \geq 1$, and that

(T3) there is an exact sequence $0 \rightarrow R \rightarrow T_{0} \rightarrow \cdots \rightarrow T_{n} \rightarrow 0$, where each $T_{i} \in \operatorname{add} T$.

The notion of tilting modules is left-right symmetric in the sense that if ${ }_{R} T$ is a tilting module of projective dimension at most $n$, then $T_{S}$, where $S=\left(\operatorname{End}_{R} T\right)^{\circ}$, is also a tilting module of projective dimension at most $n$ with $R \simeq \operatorname{End} T_{S}$. 
Dually, an $R$-module $C$ is a cotilting module of injective dimension at most $n$ if it satisfies

(C1) $\operatorname{id} T \leq n$,

(C2) $\operatorname{Ext}_{R}^{i}(T, T)=0$ for all $i \geq 1$, and that

(C3) there is an exact sequence $0 \rightarrow C_{n} \rightarrow \cdots \rightarrow C_{0} \rightarrow \mathbf{D}\left(R_{R}\right) \rightarrow 0$ with each $C_{i} \in \operatorname{add}_{R} C$.

Note that an $R$-module $T$ is a tilting module of projective dimension at most $n$ if and only if the $R^{o}$-module $\mathbf{D}\left({ }_{R} T\right)$ is a cotilting module of injective dimension at most $n$.

Lemma 2.1. Let $R$ be an Artin algebra and $T \in \bmod R$ be a tilting module of projective dimension at most $n$. Then, for any $M \in \bmod R$, there is an exact sequence $0 \rightarrow M \rightarrow U_{M} \rightarrow V_{M} \rightarrow 0$ with $U_{M} \in T^{\perp}$ and such that $V_{M}$ fits into an exact sequence $0 \rightarrow V_{M} \rightarrow T_{0} \rightarrow \cdots \rightarrow T_{n-1} \rightarrow 0$ with each $T_{i} \in \operatorname{add} T$. In particular, $V_{M}$ has projective dimension at most $n$. Moreover, $U_{M}$ can be taken as a minimal left $T^{\perp}$-approximation of $M$.

Proof. The claimed exact sequence exists by, for instance, [10, Lemma 2.2].

The following is the well-known Brenner-Butler Theorem in tilting theory (see for instance [3], 6]).

Lemma 2.2. Let $R$ be an Artin algebra and $T \in \bmod R$ be a tilting module with $S=\left(\operatorname{End}_{R} T\right)^{\circ}$. Denote $C=\mathbf{D}\left(T_{S}\right)$. Then there is an equivalence between ${ }_{R} T^{\perp}$ and ${ }_{S}^{\perp} C$, given by the functor $\operatorname{Hom}_{R}(T,-)$. Moreover, for any $U, W \in T^{\perp}$ and any $i \geq 0$, we have that $\operatorname{Ext}_{R}^{i}(U, W) \simeq \operatorname{Ext}_{S}^{i}\left(\operatorname{Hom}_{R}(T, U), \operatorname{Hom}_{R}(T, W)\right)$.

\section{Stability of generalized Auslander-Reiten conjecture}

Throughout the section, we fix $R$ to be an Artin algebra and $T \in \bmod R$ to be a tilting module of projective dimension at most $n$ with $S=\left(\operatorname{End}_{R} T\right)^{o}$. Then, for an $M \in \bmod R$, we have two modules $U_{M}, V_{M}$ associated with $T$ and $M$ as shown in Lemma 2.1.

We note first that the generalized Auslander-Reiten conjecture can be reformulated as follows.

Let $R$ be an Artin algebra and $X \in \bmod R$. If $X \oplus R \in X^{\gg 0}$, then $\operatorname{pd}_{R} X<\infty$.

It is just this version that we use in the following to refer to the generalized Auslander-Reiten conjecture.

To prove the main result, we need some lemmas.

Lemma 3.1. Let $M \in \bmod R$. Then $M \oplus R \in M^{\gg 0}$ if and only if $U_{M} \oplus R \in U_{M}^{\gg 0}$.

Proof. Consider the exact sequence $0 \rightarrow M \rightarrow U_{M} \rightarrow V_{M} \rightarrow 0$ in Lemma2.1. Since $V_{M}$ has finite projective dimension, $M \oplus R \in V_{M}^{\gg 0}$. It follows that $M \oplus R \in M \gg 0$ if and only if $M \oplus R \in U_{M}^{\gg 0}$ by applying the functor $\operatorname{Hom}_{R}(-, M \oplus R)$ to the sequence. Note that if $R \in N^{\gg 0}$ for an $R$-module $N$, then $V \in N^{\gg 0}$ for any $R$-module $V$ of finite projective dimension. Hence it also follows that $U_{M} \oplus R \in U_{M}^{\gg 0}$ if and only if $M \oplus R \in U_{M}^{\gg 0}$ by applying the functor $\operatorname{Hom}_{R}\left(U_{M},-\right)$ to the sequence. It then follows that $M \oplus R \in M^{\gg 0}$ if and only if $U_{M} \oplus R \in U_{M}^{\gg 0}$.

Lemma 3.2. Let $U \in \bmod R$. Then $T \in U^{\gg 0}$ if and only if $R \in U^{\gg 0}$. In particular, $U \oplus R \in U^{\gg 0}$ if and only if $U \oplus T \in U^{\gg 0}$. 
Proof. The conclusion easily follows from the two exact sequences in the definition of tilting modules. One is $0 \rightarrow P_{n} \rightarrow \cdots \rightarrow P_{0} \rightarrow T \rightarrow 0$ with each $P_{i}$ projective and the other is $0 \rightarrow R \rightarrow T_{0} \cdots \rightarrow T_{n} \rightarrow 0$ with each $T_{i} \in \operatorname{add}_{R} T$.

Lemma 3.3. Let $M \in \bmod R$ and let $m$ be a nonnegative integer. Then $\operatorname{pd} M<\infty$ if and only if $U_{M} \in \hat{T}$.

Proof. It follows that $\operatorname{pd} M<\infty$ if and only if $\operatorname{pd} U_{M}<\infty$ from the exact sequence $0 \rightarrow M \rightarrow U_{M} \rightarrow V_{M} \rightarrow 0$ in Lemma 2.1. Thus it is enough to show that $\operatorname{pd} U_{M}<\infty$ if and only if $U_{M} \in \hat{T}$.

It is easy to see that if $U_{M} \in \hat{T}$, then $\operatorname{pd} U_{M}<\infty$, since $\operatorname{pd} T<\infty$. Now assume that $\operatorname{pd} U_{M}<\infty$. Since $U_{M} \in T^{\perp}$, we can also deduce that $U_{M} \in \hat{T}$ from the proof of [8, Proposition 2.10].

The following proposition shows that we can transfer the generalized AuslanderReiten conjecture for $R$ to a version related to a tilting $R$-module.

Proposition 3.4. The Artin algebra $R$ satisfies the generalized Auslander-Reiten conjecture if and only if, for any $U \in T^{\perp}, U \oplus T \in U^{\gg 0}$ implies that $U \in \hat{T}$.

Proof. The if-part. Take any $M \in \bmod R$ such that $M \oplus R \in M^{\gg 0}$. Then $U_{M} \oplus T \in$ $U_{M}^{\gg 0}$ by Lemmas 3.1 and 3.2 . Hence $U_{M} \in \hat{T}$ by assumptions of the if-part. It follows that $\operatorname{pd} M<\infty$ by Lemma 3.3 .

The only-if part. Take any $U \in T^{\perp}$ such that $U \oplus T \in U^{\gg 0}$. Then $U \oplus R \in U^{\gg 0}$ by Lemma 3.2. Hence $\operatorname{pd} U<\infty$ by assumptions of the only-if part. It then follows that $U \in \hat{T}$ by Lemma 3.3 again.

We now consider $S$-modules.

Lemma 3.5. Let $S$ be an Artin algebra and $n$ be a positive integer. Then for any $M \in \bmod S, M \oplus S \in M^{\gg 0}$ if and only if $\Omega_{S}^{n} M \oplus S \in\left(\Omega_{S}^{n} M\right)^{\gg 0}$.

Proof. Consider the exact sequence $0 \rightarrow \Omega_{S}^{n} M \rightarrow S_{n-1} \rightarrow \cdots \rightarrow S_{0} \rightarrow M \rightarrow 0$ with each $S_{i}$ projective. By applying the functor $\operatorname{Hom}_{S}(-, M \oplus S)$ to the sequence, we obtain that $M \oplus S \in M \gg 0$ if and only if $M \oplus S \in\left(\Omega_{S}^{n} M\right)^{\gg 0}$. Now by applying the functor $\operatorname{Hom}_{S}\left(\Omega_{S}^{n} M,-\right)$ to the sequence, we obtain that $M \oplus S \in\left(\Omega_{S}^{n} M\right)^{\gg 0}$ if and only if $\Omega_{S}^{n} M \oplus S \in\left(\Omega_{S}^{n} M\right)^{\gg 0}$.

Proposition 3.6. Let $S$ be an Artin algebra and $C \in \bmod S$ be a cotilting module of injective dimension at most $n$. Then $S$ satisfies the generalized Auslander-Reiten conjecture if and only if, for any $X \in{ }^{\perp} C, X \oplus S \in X^{\gg 0}$ implies that $\operatorname{pd} X<\infty$.

Proof. The only-if-part is direct, so we need only to show the if part. Take any $M \in \bmod R$ such that $M \oplus R \in M^{\gg 0}$. Then $\Omega_{S}^{n} M \oplus S \in\left(\Omega_{S}^{n} M\right)^{\gg 0}$ by Lemma 3.5. Note that $\Omega_{S}^{n} M \in{ }^{\perp} C$, so $\operatorname{pd}\left(\Omega_{S}^{n} M\right)<\infty$ by assumptions of the if part. Clearly this implies that $\operatorname{pd} M<\infty$.

We are in the position to prove our main result.

Theorem 3.7. Let $R$ be an Artin algebra and $T \in \bmod R$ be a tilting module with $S=\left(\operatorname{End}_{R} T\right)^{\circ}$. Then $R$ satisfies the generalized Auslander-Reiten conjecture if and only if $S$ does as well.

Proof. The conclusion follows easily from Proposition 3.4 Lemma 2.2 and Proposition 3.6 . 
Corollary 3.8. Let $R$ be an Artin algebra and $T \in \bmod R$ be a tilting module with $S=\left(\operatorname{End}_{R} T\right)^{\circ}$. Then $R^{o}$ satisfies the generalized Auslander-Reiten conjecture if and only if $S^{o}$ does as well.

Proof. The conclusion follows from Theorem 3.7 using the fact that $T$ is also a tilting $S^{o}$-module with $R^{o} \simeq\left(\operatorname{End}_{S^{o}} T\right)^{o}[\underline{3}$.

Corollary 3.9. Let $S$ be an Artin algebra and $C \in \bmod S$ be a cotilting module with $R=\left(\operatorname{End}_{S} C\right)^{o}$. Then $S$ satisfies the generalized Auslander-Reiten conjecture if and only if $R$ does as well.

Proof. The conclusion follows from Theorem 3.7 using the fact that $T=\mathbf{D} C$ is a tilting $R$-module with $S \simeq\left(\operatorname{End}_{R} T\right)^{o}$.

\section{REFERENCES}

[1] M. Auslander and I. Reiten, On a generalized version of the Nakayama conjecture, Proc. Amer. Math. Soc. 52 (1975), 69-74. MR0389977 (52:10806)

[2] M. Auslander and I. Reiten, Applications of contravariantly finite subcategories, Adv. Math. 86 (1) (1991), 111-152. MR.1097029 (92e:16009)

[3] Y. Miyashita, Tilting modules of finite projective dimension, Math. Zeit. 193 (1986), 113-146. MR852914 (87m:16055)

[4] S. Pan and C. Xi, Finiteness of finitistic dimension is invariant under derived equivalences, Journal of Algebra 322 (2009), 21-24. MR2526372

[5] D. Happel, Reduction techniques for homological conjectures, Tsukuba J. Math. 17 (1) (1993), 115-130. MR:1233117 (94h:16015)

[6] D. Happel, Triangulated categories in the representation theory of finite-dimensional algebras, London Math. Soc. Lect. Note Ser., 119, Cambridge Univ. Press, Cambridge, 1988. MR.935124(89e:16035)

[7] H. Tachikawa, Quasi-Frobenius rings and generalizations, Lect. Notes Math., 351, SpringerVerlag, Berlin-New York, 1973. MR0349740 (50:2233)

[8] J. Wei, Finitistic dimension and restricted flat dimension, J. Algebra 320 (2008), 116-127. MR2417981 (2009e:16013)

[9] J. Wei, A note on Auslander bounds, arXiv: 0802.1085v2.

[10] J. Wei and C. Xi, A characterization of the tilting pair, J. Algebra 317 (2007), 376-391. MR2360155 (2009b:16034)

Department of Mathematics, Nanjing Normal University, Nanjing 210046, People's Republic of China

E-mail address: weijiaqun@njnu.edu.cn

Current address: Mathematisches Institut, Universität zu Koeln, Weyertal 86-90, D-50931 Koeln, Germany 